\title{
The effects of integrative healthcare on Peruvian Indigenous groups
}

\section{Rebecca Wolff}

Arts and Sciences, College of Arts, University of Guelph, Guelph, ON Canada. Faculty supervisor: Dr. Kate Parizeau. For correspondence, please email: rjwolff@hotmail.ca.

\begin{abstract}
Indigenous communities are vulnerable to a variety of health risks due to political marginalization, socioeconomic challenges and geographic isolation. Most developed and developing nations rely mainly on biomedical healthcare services, which do not adequately incorporate the use of traditional medicinal knowledge. Peru is home to over $\mathbf{5 0}$ Indigenous groups, many of which practice holistic and traditional approaches to healthcare. Peruvian healers and medicinal plants play an integral role in such traditional medicinal systems. Integrative healthcare, which incorporates Indigenous medicine into the biomedical healthcare system, is a potential solution to improving healthcare services for an entire nation. However, integrative healthcare fails to address the lack of accessibility and affordability of the Peruvian healthcare system for marginalized populations. Traditional medicine reflects a multi-dimensional, spiritual and individualized approach to healthcare that is in conflict with the scientific and esoteric nature of the biomedical system. Incorporating traditional medicine into the biomedical system could threaten the existence of traditional medicinal knowledge and decrease the need for dissemination of traditional knowledge and culture. In a Peruvian context, integrative healthcare would have a detrimental impact on the maintenance and dissemination of Indigenous Peruvian medical knowledge.
\end{abstract}

Keywords: Peru; Indigenous; health; policy; traditional, complementary and alternative medicine (TCAM)

\section{Introduction}

There are an estimated 370 million Indigenous people living throughout the world in a variety of different environments, from mountainous terrain to the Amazon (WHO 2013). Indigenous communities are often vulnerable to a variety of health risks due to political marginalization, socioeconomic challenges, and geographic isolation (Ford et al. 2010). Many Indigenous groups have sustained their traditional ways of living for centuries, including their fundamental spiritual beliefs and traditional health applications. Traditional medicine, which stems from Indigenous perceptions of health and cosmology, plays an important role for many Indigenous groups, as it is often their main form of healthcare (WHO 2013). In previous research conducted with African and South American Indigenous communities, traditional medicine was found to be the main form of healthcare in Indigenous communities due to its accessibility and affordability, and its incorporation of the holistic ideals that form the cosmology of many Indigenous groups (Vandebroek et al. 2004; Yinegar et al. 2007; Valadeau et al. 2009; Luziatelli et al. 2010; Odonne et al. 2013).

Despite the existence of strong traditional medicinal knowledge in most countries, many developed and developing nations rely mainly on biomedical healthcare, which focuses on scientific and evidence-based understanding of symptoms and causes of illness. Treatment of disease is approached using technological advances such as pharmaceuticals and medical technology (Hoenders et al. 2012).

As populations continue to grow and the deadline for achieving the 2015 Millennium Development Goals approaches, improving delivery of healthcare services and illness prevention is becoming more important within developing nations. Traditional health practitioners have been considered skilled healthcare professionals (Greene et al. 2004; Gonzales et al. 2008) and wild medicinal plants have been found to have biomedical healing properties (Valadeau et al. 2009; Luziatelli et al. 2010; Odonne et al. 2013). Incorporating traditional medicinal practices and practitioners into national healthcare systems using integrative healthcare has been proposed as a method by which countries can efficiently improve healthcare services (Pillsbury 1982; Chi 1994; Janes 1999; Gonzales et al. 2008). Biomedical and traditional health systems are completely different in application and practice. In Peru, Indigenous access and usage of biomedical services is very limited. The integration of traditional medicine into the biomedical system would not address the existing accessibility issues. 
Integration would likely mean the inherent theology of traditional medicine is lost. There could also be severe consequences for the survival of Indigenous culture as a result of integrative health strategies. By looking at Peruvian Indigenous cosmology and literature on integrative healthcare strategies, this paper will demonstrate that an integrative healthcare system in Peru would be more detrimental than beneficial for Indigenous communities.

\section{Background}

\section{Key Definitions}

In this paper, the United Nations' definitions for 'Indigenous peoples/communities/groups' and 'traditional medicine' will be used. These specific definitions have been chosen because of the United Nations' development of the Declaration on the Rights of Indigenous Peoples and their commitment to programs that protect and conserve Indigenous culture. According to the International Labor Organization Convention No.169 of the United Nations, the term Indigenous peoples applies to (UN 2004):

a) tribal peoples in independent countries whose social, cultural and economic conditions distinguish them from other sections of the national community and whose status is regulated wholly or partially by their own customs or traditions or by special laws or regulations;

b) peoples in independent countries who are regarded as indigenous on account of their descent from the populations which inhabited the country, or a geographical region to which the country belongs, at the time of conquest or colonization or the establishment of present state boundaries and who irrespective of their legal status, retain some or all of their own social, economic, cultural and political institutions.

Traditional medicine is defined as (UN 2004):

The sum total of the knowledge, skills, and practices based on the theories, beliefs, and experiences indigenous to different cultures, whether explicable or not, used in the maintenance of health as well as in the prevention, diagnosis, improvement or treatment of physical and mental illness.

\section{Geography of Peruvian Indigenous groups}

Peru is home to 59 Indigenous groups and although the country is rapidly developing due to economic development, natural resource extraction and increased infrastructural development, there are still thousands of Indigenous communities living throughout the Andean and Amazonian regions of the country (Luziatelli 2010). In this developmental context, the interactions between Indigenous communities and non-indigenous Peruvians are increasing. Their native lands are also in danger of destruction due to deforestation and resource development (Hofmeijer et al. 2012). As a result, Indigenous communities are adapting both their lifestyles and culture to fit the changing geography and infrastructure of their country.

Peru is home to Indigenous groups including the Yanesha, Chayahutia, and Ashaninka in the Amazon and Quechua in the Andes. In the deeper Amazonian regions, the climate is warmer year-round and communities live mainly on subsistence farming of cassava, plantain, beans, sugar, and peanuts (Odonne et al. 2012). Amazonian communities are typically located near a body of water such as a river, tributary or lake in order to fish, as hunting or raising livestock are practiced less frequently (Valadeau et al. 2009). Communities in the Andes live at higher altitudes in a much colder climate, and raise livestock and produce crops such as corn, potatoes, and beans for subsistence (Bastien 1983). The primary language spoken in most communities is an Indigenous language, though formal schooling in or outside of the community is conducted in Spanish (Mathez-Stiefel et al. 2011). Community structure is very similar in both the Amazon and Andes regions, as a 'junta directive,' or body of community officials led by a chief or president, controls community organizations and asserts authority over collective decision-making (Kensinger et al. 2000). Many communities also have traditional healers such as spiritual healers, herbalists or midwives. Healers are considered knowledgeable and important members of communal society (Valadeau et al. 2009).

Peru uses a decentralized health system, $60 \%$ of which is overseen by the Peruvian Ministry of Health, known as Ministerio de Salud or MINSA (WHO 2013). MINSA is divided into regional, provincial, and district level bodies. Each district has a local hospital, while many towns and communities also contain health clinics with a licensed health official (Mathez-Stiefel et al. 2011; WHO 2013). This MINSA-employed health official is responsible for visiting the clinic regularly to provide health services (MINSA 2012). Anecdotal evidence from the author's personal experience with Peruvian Quechua communities has shown that if there is no clinic within the community, residents must travel to a neighboring community or to the district hospital to receive biomedical healthcare services.

\section{Cosmology}

Peruvian Indigenous cultures are based on precolonized traditions and practices (Luziatelli et al. 2010). Although Spanish invaders attempted to enforce Western religion on colonized communities, they had very little success in the adoption of their Western beliefs (Tournon 2012). While each Peruvian ethnic group has its own story of the existence of the world, there is a common belief in an allpowerful creator. In Andean culture, it is Pachamama, otherwise known as Mother Earth, who is intrinsically tied to the natural environment (Chelala 2007). In Quechua 
communities in the Cusco region, food is never eaten until a small amount has been poured on the land to be returned to Pachamama as a sign of respect (Davidson 1983). Deities and/or spirits are seen to be responsible for the creation of the spiritual and natural worlds; as a result they are viewed with respect (Vanderbroek et al. 2004; Chelala 2007; Valadeau et al. 2009; Luziatelli et al. 2010). This connection with the natural world relates to the subsistence living of Indigenous groups and the importance that the land plays in their daily life. Subsistence communities are dependent on their natural surroundings for resources such as timber, food and medicine. Sustainable usage of these natural resources and environmental conservation is essential to ensure that this subsistence way of living is possible in the future (Hofmeijer et al. 2013). Belief in the interconnectedness of the spiritual, environmental and physical worlds also influences the nature of traditional medicine and how it is used.

\section{Traditional medicine: A holistic approach}

In both the Andean and Amazonian Indigenous cultures, traditional medicine is a holistic, multi-dimensional approach to healthcare. Unlike in the biomedical system, such as that of Western cultures, the body is not simply a physical or scientific object. Instead, the body, and health of one's body, is dependent on its relations with the spiritual, natural and physical world (Vanderbroek et al. 2004; Chelala 2007; Yinegar et al. 2007; Valadeau et al. 2009; Luziatelli et al. 2010; Odonne et al. 2013). While some illnesses are perceived to be due to biomedical causes like malaria, which results from a vector borne disease transmitted by mosquitoes, other illnesses do not have any relation to biomedical diseases or causes (Odonne et al. 2013). These illnesses are considered to be spirit-based, as they are believed to be caused by spirits or natural elements in the environment (Valadeau et al. 2009). For example, rainbow shock illness is caused by standing near a rainbow, which typically occurs during sunny periods of rain. An illness called mal aire results from bad spirits that enter the body and cause diarrhea, pain and fever (Valadeau et al. 2009; Odonne et al. 2013). Biomedical symptoms such as skin irritation and fever are often associated with spirit-based illness. The cause may be based on an individual's actions towards, or relationship with another person or the natural environment (Vanderbroek et al. 2004; Yinegar et al. 2007; Valadeau et al. 2009; Luziatelli et al. 2010; Odonne et al. 2013). Treating the land with disrespect, such as overharvesting wild plants or not honouring deities such as Pachamama, are believed to be causes of spirit-based illness (Chelala 2007).

Traditional healers play an essential role in the diagnosis and treatment of spirit-based illnesses. A healer identifies what spiritual agent is causing the disease, potentially through the use of medicinal plants, smoke baths or hallucinatory drugs (Valadeau et al. 2009; Luziatelli et al. 2010; Odonne et al. 2013). In certain Indigenous groups, becoming a healer is an intensive process that involves years of traditional learning and skill building in medicinal plant usage and traditional medicinal practices (Valadeau et al. 2009). In this context, healers are highly respected as knowledgeable members of the community (Luziatelli et al. 2010). Healers are also very important in the dissemination of traditional medicinal knowledge and culture, as they are experts in the field of traditional medicine and are often responsible for teaching newer generations (Overwalle 2005).

Medicinal plants and self-medication are key methods through which non-spiritual illnesses are treated. It is not uncommon for men, women, and children to all have some knowledge of traditional medicinal practices and of medicinal plant uses (Gold et al. 2011). Research has found that in most Indigenous communities, $75-85 \%$ of plants used in traditional medicine are wild, found outside of the home, and non-cultivated (Valadeau et al. 2009; Luziatelli et al. 2010; Mathez-Stiefel et al. 2011, 2012a, 2012b; Odonne et al. 2013). This further emphasizes why cultural beliefs in relation to the land are so important, as many Peruvian Indigenous groups are dependent on the natural growth of wild plants in order to access traditional medicine sources.

Medicinal plant knowledge is passed down to community members through familiar, horizontal and vertical dissemination pathways (Mathez-Stiefel et al. 2011). In the past, children would be selected to train under a traditional healer to specialize in areas of traditional medicine (Valadeau et al. 2009). However, this is increasingly less common as Indigenous communities become more acculturated due to market access, globalization and social changes (Valadeau et al. 2009; Hofmeijer et al. 2013). For example, the creation of community schools that teach national education curriculums has been shown to correlate with decreased dissemination of traditional medicinal knowledge (Giovanni et al. 2011). Many school programs do teach the community's Indigenous language or cultural history; however, full school days limit the amount of time students can spend learning about traditional medicine with community members (Giovanni et al. 2011). As a result of schooling and other social influences, Indigenous communities are changing and knowledge of traditional medicine is dimishing.

\section{Maintenance of traditional medicine}

The level to which a community has maintained its traditional culture and medicinal practices varies depending on its level of market integration and how it may have been affected by environmental degradation (Davidson 1983; Giovanni et al. 2011; Mathez-Stiefel et al. 2011, 2012a, 2012b; Sato 2012). With the expansion of development in both the Andean and Amazonian regions of Peru, more communities are connected to urban marketplaces, thus resulting in decreased geographic isolation (Mathez-Stiefel et al. 2011, 2012a, 2012b; Reyes-Garcia et al. 2013). This is changing the nature of Indigenous communities from subsistence farming to cash crop farming and wage-paying employment (Reyes-Garcia et al. 2013). Having monetary 
income and improved access to urban centers is changing the role that traditional ways of life play within the community. Monetary income means that it is more economically feasible to purchase goods that are sold at stores or markets, such as Western clothing, packaged foods, and household goods. The author's anecdotal experience with the Shipibo in the Peruvian Amazon showed that many community members have stopped wearing traditional dress and few people remember how to prepare a traditional cassava-based beverage called masato.

It is not uncommon for Indigenous male family members to seek wage-based employment outside the community in larger urban areas (Mathez-Stiefel et al. 2011, 2012a, 2012b). This impacts the household's reliance on farming as its primary means of living. Employment in wage-labor, or the need for cash crops, has also led to increased environmental degradation of local resources. Both legal and illegal logging companies are degrading local forest areas, leading to rapid destruction of the forest and wildlife (Hofmeijer et al. 2013). It is becoming more difficult to find wild plants normally used in conducting traditional medicine, such as in Chayahuita communities in the Peruvian Amazon where illegal logging is destroying medicinal trees (Hofmeijer et al. 2013). Medicinal knowledge is lessening as certain species no longer exist and information about these plants can no longer be passed down to future generations (Hofmeijer et al. 2013).

The variety of different threats to both traditional knowledge and medicinal plants in Peruvian Indigenous communities poses a huge concern for maintaining cultural practices. This makes communities more vulnerable to the negative consequences that integrative healthcare could have on Indigenous knowledge and traditions.

\section{The integrative healthcare strategy}

Integrative healthcare can be defined as a practice of medicine that incorporates available therapeutic approaches, including traditional medicine, healing, and other forms of alternative or holistic medicine, into an existing biomedical healthcare approach (Hoenders et al. 2012). According to the World Bank document titled Investing in World Health, increased healthcare services are worthy of public investment (Janes 1999). Finding ways in which to fund new healthcare programs or increase outreach of health services can be challenging for developing countries whose economies are still strengthening and may require that monetary resources be allocated to other areas (Chi 1994; Janes 1999; Gonzales et al. 2008). Importing foreign resources or aid to increase health care will result in dependence on external donors or in future debt. In contrast, traditional medicine can be viewed as a self-reliant, self-sufficient resource (Chi 1994; Gonzales et al. 2008) that is already used by a large proportion of rural and Indigenous populations.

A hypothetical integrative health care model in Peru would incorporate aspects of traditional medicine into the existing healthcare program offered by the Ministry of Health to increase services offered at hospitals and clinics.
For example, health professionals working at local clinics (typically located in larger towns or urban centers) could prescribe medicinal plants instead of pharmaceuticals and traditional healers could be trained and employed to work at local clinics and hospitals.

\section{Benefits of an integrative healthcare system}

At the community level, the primary benefit of an integrative healthcare system is that it promotes increased usage of traditional medicinal resources that are already trusted and practiced by Indigenous communities (Neba 2011). In communities that have had poor experiences with the existing biomedical system, such as failed recovery with pharmaceuticals or bad relationships with clinical physicians, traditional medicine is often seen as more affordable, accessible and legitimate (Neumann et al. 1982; MathezStiefel et al. 2011). In this situation, offering traditional medicinal services might make these particular communities feel more comfortable seeking out national healthcare services (Neumann et al. 1982). Alternatively, traditional healers could play the role of government-recognized community healthcare workers. An example of this type of integrative strategy can be seen in traditional birth assistant (TBA) programs in Ghana and the Philippines. Selected traditional midwives from Indigenous communities are trained as recognized birth attendants and community sexual health promoters (Neumann 1982). In a similar fashion, traditional healers could be selected, trained and incorporated into the Peruvian medical system to provide community level treatment and assume roles in government health clinics. This could increase access to healthcare at the community level.

A key component of implementing an integrative healthcare system is a focus on extensive training and communication between healthcare professionals. This helps to ensure that professionals from both systems perform well within an integrative setting. In Peru and worldwide, there is still a stigma towards the legitimacy of Indigenous traditional medicine as a healing practice (Bastien 1991, Sato 2012). Creating dialogue between biomedical doctors and traditional healers could be incredibly beneficial in helping biomedical practitioners to better understand the holistic and interpersonal concepts of traditional medicine (Kaboru et al. 2006). For traditional healers, being part of an integrated system would require training to help standardize available medicinal plant treatments and other traditional practices (Kaboru et al. 2006). Training provides an opportunity to teach healers about specific scientific technologies that they could functionally incorporate into their health systems, such as recognizing illness that must be treated by pharmaceuticals (Bastien 1982). This could improve the performance of healers as providers of mental and physical health resources within Indigenous communities.

It is important to note that while integrative healthcare is sometimes interpreted as a means to better incorporate Indigenous culture into national policies, the intent of integrative health systems is to increase the affordability of 
healthcare services for the country involved (Kaboru 2006). While Indigenous groups are highly marginalized and at risk for a variety of health issues, they are not the only population intended to benefit from an integrative system (Ford et al. 2010). Integrated healthcare should positively impact the entire country, although it is often assumed Indigenous groups will benefit most.

\section{Discussion}

\section{Existing issues within the biomedical healthcare system}

Integrative healthcare would be more beneficial for Indigenous groups if biomedical services were desired and used consistently. Research shows that presently, Indigenous groups prefer self-medication and visits to traditional healers over biomedicine. This is due to issues with the accessibility and affordably of biomedical care, and the methods and philosophy of the biomedical healthcare system (Giovanni et al. 2011; Mathez-Stiefel et al. 2011, 2012a, 2012b).

During the author's experience working with Peruvian Quechua groups, it was observed that if a community did not have a local clinic to receive biomedical healthcare, people would have to travel to the nearest clinic or hospital, which could take several hours by vehicle. Without a personal means of transport, it is necessary to pay for public transportation, a cost that is often unaffordable based on the socioeconomic status of the afflicted patient and their family (Day 2013). If a household does not have monetary income due to its reliance on subsistence farming, it may have to sell its own harvested goods in order to pay for a visit to a hospital or clinic. This puts the household at further health risk due to depleted personal food stores (Bastien 1982).

Affordability and accessibility of healthcare are closely related. This includes issues such as travel time to clinics, the monetary cost of this travel and the effect of travelling on the family (i.e., decreased time for daily activities and farming). This is an important issue for the current Peruvian biomedical system that needs to be addressed. In communities where national healthcare services are not easily available, the role of traditional medicine as the main form of healthcare becomes even more important and necessary (Mathez-Stiefel et al. 2012b). Integrating traditional medical practices into local clinics without addressing these concerns makes an integrative system less effective for Indigenous communities. Their need for improved access to health services will not have changed; yet, protection of their traditional medicinal practices may be compromised through widespread usage of these cultural traditions in clinics across the country.

Research has shown that Indigenous communities prefer traditional medicine to biomedical alternatives because of the non-holistic nature of the biomedical health system's approach to patient care (Gold et al. 2011; Mathez-Stiefel et al. 2011, 2012b). In Indigenous Andean communities, many people believe that pharmaceuticals and other biomedical treatments lessen symptoms but do not actually treat illness, something that can only be done by medicinal plants. This belief correlates strongly with the cosmology of many Indigenous Peruvian cultures: that the body is not only physical but also connected to the spiritual and natural worlds (Chelala 2007). In contrast, the biomedical health system is based on esoteric knowledge rather than traditional knowledge and beliefs (Hollenburg 2006). Biomedicine tends to be more broadly concerned with clinically based issues, such as treating patients for only symptoms present at the time without looking at their spiritual or emotional history (Hollenburg 2006). A variety of well-documented, scientific techniques such as pharmaceuticals, blood tests and $\mathrm{x}$-rays are used to diagnose illness and prescribe proper treatment (Barbee 1986). As a result of these extreme differences, biomedical services are highly incompatible with traditional medicine, which further influences Indigenous communities to avoid using the biomedical system.

Another concern with the biomedical system involves the patient going to an unknown practitioner. Unlike biomedical doctors, traditional healers are not strangers to their patients; they are trusted and respected members of the community (Bastien 1982; Kale 1995). They also allow, and often encourage, the community and family to take part in the healing process by being present during diagnosis, treatment, and by providing feedback and support (Kale 1995). Illness is therefore never just the burden of the patient alone. A network made up of the healer and community supports a patient, and healing is a highly interpersonal experience that reflects the spiritual and emotional needs of each patient. In general, the biomedical system is much less personal and the outcome, not the physician-patient relationships, matter in terms of treating illness (Hollenburg 2006). Incorporating traditional medicine into the existing health system could threaten the philosophical fundamental elements of traditional medicine, specifically how patients are personally treated and the role of the healer in helping them. These intricate relationships between healer, patient and community could be lost, which could be detrimental to Indigenous communities and the maintenance of their medicinal traditions.

\section{Traditional vs. biomedical healthcare: A practical and theoretical challenge}

The fundamental values and applications of the traditional and biomedical health systems are intrinsically different in nature. In Peru, this presents one of the biggest challenges for creating an integrative healthcare system, as it does not seem that the two systems are compatible. Furthermore, research illustrates that Indigenous communities are skeptical of the benefits of biomedical treatments since they do not coincide with their ideas of how illness should be treated and what health consists of (Boffa et al. 2004; Graham et al. 2010; Mathez-Stiefel et al. 2011, 2012a, 2012b). The biomedical healthcare system is intrinsically different in how it provides one-on-one 
treatment to patients. Adding traditional medicinal elements to this system does not make the philosophy or goals of biomedicine more spiritual or multi-dimensional. Integrative healthcare seeks to provide more resources by which to treat illness, but in order to be truly effective, it needs to also address the differences in methodology and beliefs upon which each health system is based. Without bridging this gap, integrative health services will not address the spiritual, personal and communal aspects of traditional medical delivery that are currently fundamental aspects of Indigenous medicine.

Additionally, incorporating components of traditional medicine into the biomedical system could threaten the existence of traditional medicine knowledge and its usage. Knowledge of the property rights of traditional medicinal practices specific to certain communities or Indigenous groups would be lost and community-level dissemination of traditional medicinal knowledge would decrease.

\section{Integration as a threat to Indigenous knowledge}

Traditional health is an integral part of Indigenous culture and represents Indigenous spiritual beliefs and cosmology. In an ever-modernizing landscape, the dissemination of Indigenous culture is already challenged from acculturation, environmental degradation and market integration (Davidson 1983; Giovanni et al. 2011; MathezStiefel et al. 2011, 2012a, 2012b). Integrative medicine, and the addition of traditional medicine practices into the biomedical health systems, will further diminish the role of these procedures.

Incorporating medicinal plants into the biomedical health system could be viewed as a legal breach of rights for Indigenous groups. Knowledge of medical plants should be considered the intellectual property of the community in which the practice originated (Greene 2004). Some medicinal plant knowledge for more spiritual treatments is kept secret for ritualistic healing purposes (Kaboru 2006). Through integration, the privacy and specialty of these practices would be lost and could even result in a loss of their cultural significance. The usage of traditional medicinal plants and secretive medicinal practices without proper individual and community consent is an ethical and legal dilemma that could destroy the cultural integrity of Indigenous communities. Self-medication with wild medicinal plants still occurs within the home, implying that traditional medical knowledge has been maintained in many Indigenous communities (Mathez-Stiefel et al. 2011, 2012a, 2012b). The use of medicinal plants as a prescriptive solution in health clinics would diminish the need for the majority of community members to be taught the skills to collect, identify and learn about wild medicinal plants for selfmedication since plant remedies would be readily available in clinics or commercial settings. The dissemination and generational transmission of this knowledge would be negatively impacted, as it would no longer be necessary to collect plants for self-treatment if they could be prescribed and dispensed at local clinics. This would be detrimental to the practice of using medicinal plants in Indigenous culture, as once this knowledge is lost it is gone forever.

The holistic and multi-dimensionality of Indigenous medicinal practices could be endangered by an integrative healthcare system. Aspects of traditional medicine would have to be regulated and standardized so that they could be included within national healthcare clinics (Kale 1995; Ragunathan et al. 2010; Neba 2011). While this standardization is needed to ensure equal and safe care for all patients, it opposes the values of traditional health (Ragunathan et al. 2010). Uses of medicinal plants and the advice of a healer can no longer reflect a patient's personal relationship with the spiritual, physical and environmental worlds, as a clinical physician may not be privy to this information or focus their care on these principles. It also implies that the relationship between healer and patient will be less interpersonal, as healers may not be from a patient's community. Standardizing traditional medicine is contrary to the idea that healing and treatments should reflect the patient's personal spiritual and physical connection to their natural environment and their community. Not only is this belief essential to traditional health, but also to Indigenous cultural autonomy, cosmology, and culture. Thus, integrative medicine threatens the core nature of certain Indigenous traditions and could have detrimental effects on the maintenance of these practices in the future.

\section{Conclusion}

Integrative healthcare systems will have adverse impacts on the maintenance and dissemination of Indigenous Peruvian medicinal knowledge. The biomedical and traditional health systems function based on opposing ideologies and philosophies of how healthcare should be approached, making them highly incompatible. Biomedicine is based on esoteric knowledge and uses one-dimensional, non-personal treatment of illness (Hollenburg 2006). In contrast, traditional medicine reflects Indigenous beliefs that health is based on a patient's personal relationships with the spiritual, environmental and physical worlds (Valadeau et al. 2009; Luziatelli et al. 2010; Odonne et al. 2013). Due to these strong spiritual beliefs, traditional medicine and healers are an integral part of Indigenous culture and cosmology in Peru. Given the inaccessibility of existing biomedical services for Andean and Amazonian communities, integrating traditional resources into biomedical health clinics will not improve the availability of healthcare services for Indigenous communities. Instead, it may only increase the speed at which traditional Indigenous knowledge and culture is being changed with globalization.

Current research on traditional medicine in Peru has identified that biomedical and traditional systems can coexist without dangerous effects to Indigenous culture and medicinal practices (Mathez-Stiefel et al. 2011, 2012b). However, this research has not adequately identified the risks of an integrative strategy. More work is required to determine if any integrative health plans in South America 
have been successful at the community level and if so, how danger to Indigenous groups can be avoided or minimized. At a policy level, more information is needed on how Peru is currently trying to address issues in its existing healthcare system, and if this alone would be enough to dramatically improve healthcare services in the country.

\section{Acknowledgements}

Thank you to my faculty supervisor Kate Parizeau. This project was supported by resources from the Indigenous Health Adpatation to Climate Change (IHACC) Project.

\section{References}

Baldwin MR, Yori PP, Ford C, Moore DAJ, Gilma RH, Vidal C, Ticona E, Evans CA. 2004. Tuberculosis and nutrition: disease perceptions and healthseeking behavior of household contacts in the Peruvian Amazon. International Journal of Tuberculosis and Lung Disease. 8(12):1484-1491.

Barbee EL. 1986. Biomedical resistance to ethnomedicine in Botswana. Social Science \& Medicine. 22(1): 75-80.

Bastien JW. 1982. Exchange between Andean and Western medicine. Social Science \& Medicine. 16(7):795-803.

Bishaw M. 1991. Promoting traditional medicine in Ethiopia: a brief historical review of government policy. Social Science \& Medicine. 33(2):193-200.

Boffa J, King M, McMullin K, Long R. 2011. A process for the inclusion of Aboriginal People in health research: lessons from the Determinants of TB transmission project. Social Science \& Medicine.72(5):733-738.

Chelala C. 2007. Health in the Andes. Americas. 59(4): 54-57.

Chi C. 1994. Integrating traditional medicine into modern health care systems: examining the role of Chinese medicine in Taiwan. Social Science \& Medicine. 39(3):307-321.

Davidson, J. 1983.The survival of traditional medicine in a Peruvian barrida. Social Science \& Medicine. 17(17):1271-1280.

Day J. 2013. Sustainability and accessibility of healthcare in times of austerity: improved health promotion and transportation remain essential. Scottish Universities Medical Journal. 2(1):8-12.

Ford J, Berrang-Ford L. 2010. Vulnerability and adaptation of indigenous peoples to the health effects of climate change: a vulnerability framework. Montreal (QC): Dept. of Geography, McGill University.
Giovanni P, Reyes-Garcia V, Waldstein A, Heinrich M. 2011. Do pharmaceuticals displace local knowledge and use of medicinal plants? Estimates from a crosssectional study in a rural Indigenous community, Mexico. Social Science \& Medicine. 72(6):982-936.

Gold CL, Clapp RA. 2011. Negotiating health and identity: lay healing, medicinal plants and indigenous healthscapes in Highland Peru. Latin American Research Review. 46(3): 94-114.

Gonzales FG, Aguilar J, Villar M. 2008. World summit of harmonization on traditional, alternative and complementary medicine (TACM) in Lima, Peru. Evidence-Based Complementary and Alternative Medicine (eCAM). 7(2):271-275.

Graham H, Stamler LL. 2010. Contemporary perceptions of health from an Indigenous (Plains Cree) perspective. Journal of Aboriginal Health. 6(1):6-17.

Greene S. 2004. Indigenous people incorporated? Culture as politics, culture as property in pharmaceutical bioprospecting. Current Anthropology. 45(2):211-237.

Hoenders RHJ, Appelo MT, De Jong JTVM. 2012. Integrative Medicine: a bridge between biomedicine and alternative medicine fitting the spirit of the the age. Sociology Mind. 2(4):441-446.

Hollenburg D. 2006. Uncharted ground: patterns of professional interaction amongtcontemporary/alternative and biomedical practitioners in integrative health care settings. Social Science \& Medicine. 62(3):731-744.

Hofmeijer I, Ford JD, Berrang-Ford L, Zavaleta C, Carcamo C, Llanos E, Carhuaz C, Edge V, Lwasa S, Namanya D. 2013. Community vulnerability to the health effects of climate change among Indigenous populations in the Peruvian Amazon: a case study from Panaillo and Nuevo Progreso. Mitigation and Adaptation Strategies for Global Change. 18(7):957-979.

Janes CR. 1999. The health transition global modernity and crisis of traditional medicine: the Tibetan case. Social Science \& Medicine. 48(12):1803-1820.

Kaboru BB, Falkenberg T, Ndulo J, Muchimba M, Solo K, Faxelid E. 2006. Communities' views on prerequisites for collaboration between modern and traditional health sectors in relation to STI/HIV/AIDS care in Zambia. Health Policy. 78(2-3):330-339.

Kale R. 1995. Traditional healers in South Africa: a parallel health system. British Medical Journal. 310(6988):11821185.

Kensinger KM, Dole GE, Morin F. 2002. Guíaetnográfica de la alta Amazonia. Volumen II: Cashinahua. Quito (EC): Smithsonian Tropical Research Institute \& Instituto Francés de Estudios Andinos \& Abya-Yala. 372 p. 
Kulman KC, Richmond CAM. 2011. Addressing the persistence of tuberculosis among the Canadian Inuit population: the need for social determinants of health framework. The International Indigenous Policy Journal. 2(1):1-16.

Luziatelli G, Sorensen M, Theilade I, Molgaard P. Ashaninka medicinal plants: a case study from the native community of Bajo Quimiriki, Junin Peru. Journal of Ethnobiology and Ethnomedicine. 6(21):1-23.

Mathez-Stiefel SL, Vandebroek I. 2011. Distribution and transmission of medicinal plant knowledge in the Andean Highlands: a case study from Bolivia and Peru. Evidence-Based Complementary and Alternative Medicine (eCAM). 2012(2012):1-18.

Mathez-Stiefel SL, Brandt R, Lachmuth S, Rist S. 2012a. Are the young less knowledgable? Local knowledge of natural remedies and its transformation in the Andean Highlands. Human Ecology. 40(6):909-930.

Mathez-Stiefel SL, Vandebroek I, Rist S. 2012b. Can Andean medicine co-exist with biomedical healthcare? A comparison of two rural communities in Peru and Bolivia. Journal of Enthobiology and Ethnomedicine [Internet]. [cited $20148 \mathrm{Dec}$; 8(26). Available from: http://www.ethnobiomed.com/content/8/1/26

[MINSA] Minsterio de Salud. 2012. Aportes metodológicos para la elaboración del análisis de situación de salud de los pueblos indígenas de la Amazonia peruana. Lima (PE): Minesterio de Salud. Report No. 956-2012.

Neba EN. 2011. Traditional health care system and challenges in developing ethnopharmacology in Africa: example of Oku, Cameroon. Studies on Ethno-medicine. 5(2):133-139.

Neumann AK, Lauro P. 1982. Ethnomedicine and biomedicine linking. Social Science \& Medicine. 16(21):1817-1824.

Odonne G, Valadea C, Alban-Castillo J, Stien D. 2013. Medical etnobiography of the Chayahuita of the Paranapura basin (Peruvian Amazon). Journal of Ethnopharmacology. 146(1):127-153.

Overwalle VG. 2005. Protecting and sharing biodiversity and traditional knowledge: holder and user tools. Ecological Economics. 53(4):585-607.

Pillsbury BLK. 1982. Policy and evaluation perspectives on traditional health practitioners in national health care systems. Social Science \& Medicine. 16(21):1825-1834.

Ragunathan M, Tadesse H, Tujuba R. 2010. A crosssectional study on the perceptions and practices of modern and traditional health practitioners about traditional medicine in Dembia district, North Western Ethiopia. Pharmacognosy. 6(21):19-25.
Reyes-Garica V, Gueze M, Luz AC, Paneque-Galvez J, Macia MJ, Otra-Martinez M, Pino J, Rubio-Campillo X. 2013. Evidence of loss of traditional knowledge Bolivia. Evolution and Human Behavior. 34(4):249-257.

Sato A. 2012. Does socio-economic status explain use of modern and traditional health care services? Social Science \& Medicine.75(8):1450-1459.

Tournon J. 2002. La merma mágica. Lima (PE): Centro Amazónico de Antropología y Aplicación Practica. 459 p.

[UN] United Nations. 2004. The concept of Indigenous peoples. New York (New York): United Nations. 4 p.

Valadeau C, Castillo AJ, Sauvain M, Lores AF. 2009. The rainbow hurts my skin: medicinal concepts and plants used by the Yanesha, an Amazonian Peruvian ethnic group. Journal of Ethnopharmacology. 127(2010): 175-92.

Vandebroek I, Damme PV, Puyvelde LV, Arrazola S, Kimpe ND. 2004. Comparison of traditional healers knowledge in Bolivian Andes and Amazon. Social Science \& Medicine. 59(4):837-849.

[WHO] World Health Organziation. 2013. Country Responses: Peru [WHO Media Centre]. World Health Organization; [cited 2013 Dec 8]. Available from: http://www.who.int/workforcealliance/countries/per/en/i $\underline{\text { ndex.html }}$

[WHO] World Health Organziation. 2007. Health of Indigenous People [WHO Media Centre]. World Health Organization; [cited 2013 Dec 8]. Available from: http://www.who.int/mediacentre/factsheets/fs326/en/ind ex.html

Yinegar H, Yewhalaw D. 2007. Traditional medicinal plant knowledge and use by local healers in Sekoru District, Jimma Zone, Southwestern Ethiopia. Journal of Ethnobiology and Ethnomedicine. 3(24):24-31 